\title{
A dissipative quantum reservoir for microwave light using a mechanical oscillator
}

\author{
L. D. Tóth, ${ }^{1, *}$ N. R. Bernier, ${ }^{1, *}$ A. Nunnenkamp, ${ }^{2}$ A. K. Feofanov, ${ }^{1, \dagger}$ and T. J. Kippenberg ${ }^{1,}$ \\ ${ }^{1}$ Institute of Physics, École Polytechnique Fédérale de Lausanne (EPFL), CH-1015 Lausanne, Switzerland \\ ${ }^{2}$ Cavendish Laboratory, University of Cambridge, Cambridge CB3 OHE, United Kingdom
}

\begin{abstract}
Engineered dissipation can allow to prepare the quantum states of atoms, ions, or superconducting qubits. This is achieved with a suitably engineered coupling to a dissipative cold reservoir usually formed by an electromagnetic mode. Similarly, in the field of cavity electro- and optomechanics, the electromagnetic cavity naturally serves as a cold reservoir for the mechanical mode. Breaking from this paradigm, here we realize the opposite scenario and engineer a mechanical oscillator cooled close to its ground state into a cold dissipative reservoir for microwave photons in a superconducting circuit. By tuning the coupling to this dissipative mechanical reservoir, we demonstrate dynamical backaction control of the microwave field, leading to stimulated emission and maser action. Moreover, the reservoir can function as a useful quantum resource, allowing to implement a near-quantum-limited phase-preserving microwave amplifier. Such engineered mechanical dissipation extends the toolbox of quantum manipulation techniques of the microwave field and constitutes a novel ingredient for optomechanical protocols.
\end{abstract}

Dissipation can significantly affect the quantum behaviour of a system and even completely suppress it 1 . However, if carefully constructed, dissipation can relax the system of interest to a desired target quantum state. This pioneering insight was originally theoretically conceived and studied in the context of trapped ions [2, experimentally first realized with trapped atomic ensembles [3] and later with trapped ions [4]6. Moreover, reservoir engineering has recently also been realized in the context of circuit QED [7] 9 . In these experiments the optical or microwave field provides a dissipative reservoir to the quantum systems. In cavity optomechanics 10, in which a mechanical oscillator and electromagnetic degree of freedom are parametrically coupled, analogous ideas have been developed and reservoir engineering for the preparation of squeezed mechanical states has been theoretically proposed [11, 12 and recently demonstrated [13 15]. As in the atomic physics case, the electromagnetic field acts as the engineered environment of the quantum system of interest.

In contrast, recent theoretical work [16 20] has considered the opposite scenario where the mechanical degree of freedom is employed to provide a dissipative, cold bath for light. This engineered bath can then be employed to achieve desirable quantum states of light or to modify the optical field properties. For example, such a dissipative reservoir for light can be exploited for amplification [17, 18, entanglement generation [16] or dissipative squeezing of electromagnetic modes [19]. Moreover, it provides an ingredient to realize nonreciprocal devices 20] such as isolators, circulators or directional microwave amplifiers. For a sufficiently cold dissipative mechanical reservoir, nonreciprocal devices implemented in this manner can operate in the quantum regime, with minimal added noise.

\footnotetext{
${ }^{*}$ L. D. Tóth and N. R. Bernier contributed equally to this work.

$\dagger$ alexey.feofanov@epfl.ch

$\ddagger$ tobias.kippenberg@epHl.ch
}

Here we engineer a mechanical oscillator into a quantum reservoir for microwave light. This is achieved in a microwave optomechanical system [21] by engineering the mechanical dissipation rate to exceed that of the electromagnetic mode. This regime allows to demonstrate dynamical backaction 22, on microwave light, and the control of a microwave mode by tuning its coupling to the reservoir. Backaction amplification leads to stimulated emission of microwaves and maser action using the mechanical oscillator as the gain medium. Below the masing threshold, we implement a large-gain phasepreserving amplifier that operates with added noise 0.87 quanta (or a factor 2) above the quantum limit. Critically, this demonstrates that the mechanical reservoir for light can function as a useful quantum resource.

\section{A. Optomechanical circuit with dark and bright modes}

We utilize a scheme in which two microwave modes are coupled to the same mechanical oscillator [18]. One (auxiliary) electromagnetic mode is used to damp the oscillator via optomechanical sideband cooling [23, 24] and engineer it into a cold bath for the other (primary) electromagnetic mode (Fig. 1A). A key ingredient for the scheme is an optomechanical cooling rate of the auxiliary mode which greatly exceeds the electromagnetic decay rate of the primary microwave mode, necessitating vastly different decay rates of the employed microwave cavities. This is challenging to achieve with previously realized dual-mode circuits [15, since any parasitic coupling between the two modes opens a decay channel, equilibrating their decay in energy. Here, we address this challenge by engineering hybridized modes with inherently dissimilar decay rates arising from interference in the output channel (cf. Fig. 1B, C and SI).

Specifically, we design an electromechanical circuit using two LC resonators both coupled inductively to a common feedline, one of which has a mechanically compli- 
A

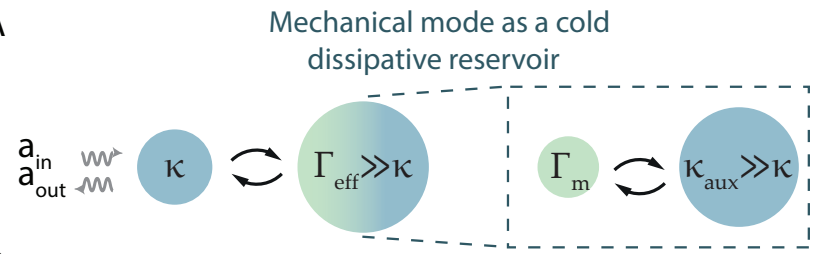

B

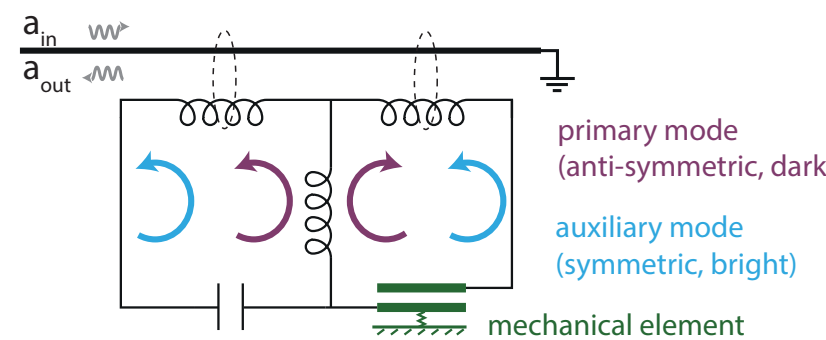

C

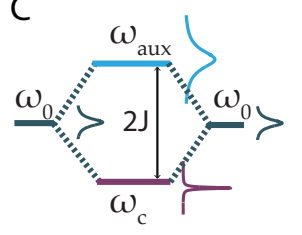

D

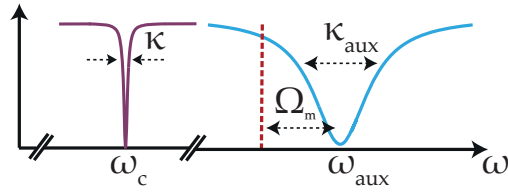

FIG. 1. Realization of a cold, dissipative reservoir for microwave light in circuit optomechanics. A. Schematic representation of a multi-mode electromechanical system in which a microwave mode with energy decay rate $\kappa$ is coupled to an engineered mechanical mode acting as a cold, dissipative reservoir with an effective energy decay rate $\Gamma_{\text {eff }}$ much greater than $\kappa$. B. Circuit realization of the electromechanical system. Two lumped-element LC circuits - one containing a mechanically compliant capacitor - with matching resonance frequencies are inductively coupled and show normal-mode splitting, forming dark and bright modes (used as the primary and auxiliary modes, respectively) to achieve $\kappa_{\text {aux }} \gg \kappa$. C. Visual representation of the mode structure and the resulting asymmetric dissipation rates, originating from the interference in the output coupling. D. Optomechanical sideband cooling the mechanical mode with the auxiliary, bright mode realizes a cold, dissipative mechanical reservoir for the primary, dark mode.

ant vacuum-gap capacitor [25] coupling mechanical vibrations to the microwave mode. The two resonators are strongly coupled through sharing a common inductor (cf. Fig. 1B). In terms of the annihilation operators $\hat{a}_{1}$ and $\hat{a}_{2}$ of the bare modes, the resulting interaction Hamiltonian is given by

$$
\hat{H}_{\mathrm{int}}=\hbar J\left(\hat{a}_{1}^{\dagger} \hat{a}_{2}+\hat{a}_{2}^{\dagger} \hat{a}_{1}\right)+\hbar \tilde{g}_{0} \hat{a}_{1}^{\dagger} \hat{a}_{1}\left(\hat{b}+\hat{b}^{\dagger}\right),
$$

where $\hat{b}$ designates the annihilation operator for the mechanical mode, $J$ the intermode coupling strength, and $\tilde{g}_{0}$ the vacuum electromechanical coupling strength to the first mode ( $\hbar$ is the reduced Planck's constant). The symmetric and antisymmetric superpositions of the bare modes $\hat{a}_{\mathrm{s}, \mathrm{a}}=\frac{1}{\sqrt{2}}\left(\hat{a}_{1} \pm \hat{a}_{2}\right)$ diagonalize the intermode coupling $J$. In terms of these hybridized modes the interac- tion Hamiltonian is given by

$$
\hat{H}_{\mathrm{int}}=\hbar J\left(\hat{a}_{\mathrm{s}}^{\dagger} \hat{a}_{\mathrm{s}}-\hat{a}_{\mathrm{a}}^{\dagger} \hat{a}_{\mathrm{a}}\right)+\hbar \frac{\tilde{g}_{0}}{2}\left(\hat{a}_{\mathrm{s}}^{\dagger} \hat{a}_{\mathrm{s}}+\hat{a}_{\mathrm{a}}^{\dagger} \hat{a}_{\mathrm{a}}\right)\left(\hat{b}+\hat{b}^{\dagger}\right) .
$$

If the bare modes are degenerate, the eigenmodes have an energy difference of $2 \hbar J$ (Fig. $1 \mathrm{C}$ ) and are now, as a result of the interaction, both coupled to the micromechanical oscillator [26] with half the bare vacuum electromechanical coupling strength $\tilde{g}_{0}$. We consider here the limit of a coupling large compared to the mechanical resonance frequency, $J \gg \Omega_{\mathrm{m}}$, implying that we can neglect the cross terms $\hat{a}_{\mathrm{s}}^{\dagger} \hat{a}_{\mathrm{a}}\left(\hat{b}+\hat{b}^{\dagger}\right)$ and other terms which are not resonant. Critically, the new eigenmodes will have dissimilar decay rates and form a bright (strongly coupled) and a dark (weakly coupled) mode resulting from interference of the bare-mode external coupling rates $\kappa_{1}^{\mathrm{ex}}, \kappa_{2}^{\mathrm{ex}}$ to the output channel (cf. SI). The symmetric, bright mode interferes constructively, leading to an external energy decay rate to the output feedline of $\kappa_{\mathrm{s}}^{\mathrm{ex}}=\kappa_{1}^{\mathrm{ex}}+\kappa_{2}^{\mathrm{ex}}$. Whereas, the antisymmetric, dark mode interferes destructively, leading to a decreased external coupling rate $\kappa_{\mathrm{a}}^{\mathrm{ex}}=\left|\kappa_{1}^{\mathrm{ex}}-\kappa_{2}^{\mathrm{ex}}\right|$. Physically, one can understand the difference by considering the topology of current flow in the modes (cf. Fig. 1B). The symmetric mode has current flowing in the same direction in both resonators, causing their external magnetic flux to create currents that add up, leading to an increased coupling rate to the feedline. The antisymmetric mode has current flowing in opposite directions in the two resonators, causing the external magnetic flux to create currents that cancel out, leading to a suppression in the external coupling to the feedline (cf. Fig. 1B, C and SI). For bare coupling rates similar in magnitude $\left(\kappa_{1}^{\mathrm{ex}} \approx \kappa_{2}^{\mathrm{ex}}\right)$, this enforces the coupling-rate hierarchy $\kappa_{\mathrm{a}}^{\mathrm{ex}} \ll \kappa_{\mathrm{s}}^{\mathrm{ex}}$ necessary to achieve a dissipative mechanical reservoir with the present scheme [18. In the remaining of the letter, we refer to the dark mode as the primary mode and the bright mode as the auxiliary mode with resonance frequencies $\omega_{\mathrm{c}}$ and $\omega_{\mathrm{aux}}$ and energy decay rates $\kappa$ and $\kappa_{\text {aux }}$, respectively (Fig. 11).

\section{B. Realization of a dissipative mechanical reservoir}

We realize the electromechanical circuit experimentally by fabricating two lumped-element LC circuits coupled to each other via a common inductor, made from thin-film aluminium on a sapphire substrate (cf. SI for the details of fabrication, design and full circuit parameters). The primary and auxiliary modes have resonance frequencies $\left(\omega_{\mathrm{c}}, \omega_{\text {aux }}\right)=2 \pi \cdot(4.26,5.48) \mathrm{GHz}$ with total energy decay rates $\left(\kappa, \kappa_{\text {aux }}\right)=2 \pi \cdot(118,4478) \mathrm{kHz}$, respectively (thus, $\kappa_{\text {aux }} / \kappa \approx 38$ ). This clear hierarchy in the energy relaxation rates indeed originates from the vastly dissimilar engineered external coupling to the feedline, with $\kappa_{\mathrm{ex}}=2 \pi \cdot 42 \mathrm{kHz} \approx 1 \%$ of $\kappa_{\mathrm{aux}}^{\mathrm{ex}}$. The mechanical resonator is a parallel-plate capacitor with a suspended top electrode, having a resonance frequency of the fundamental flexural mode $\Omega_{\mathrm{m}}=2 \pi \cdot 5.33 \mathrm{MHz}$ and a decay rate 
$\Gamma_{\mathrm{m}}=2 \pi \cdot 30 \mathrm{~Hz}$. This mechanical mode couples to both the primary and auxiliary modes with a vacuum electromechanical coupling strength $g_{0}=\tilde{g}_{0} / 2=2 \pi \cdot 60 \mathrm{~Hz}$. Details of the calibration procedure are described in Supplementary Information. Importantly, the resolved sideband regime is still attained for both microwave modes, i.e. $\Omega_{\mathrm{m}}>\kappa_{\text {aux }} \gg \kappa$. Fig. 2 A, B show an optical image of the fabricated circuit and a scanning electron micrograph of the drum-type capacitor, respectively. The simplified measurement setup is shown in Fig. 2 C. In brief, the device is mounted on the base plate of a dilution refrigerator and cooled to a base temperature of ca. 10 $\mathrm{mK}$. The microwave input lines are heavily attenuated to suppress residual thermal noise and, in addition, filter cavities are employed to remove unwanted frequency noise from the applied tones (cf. SI). After amplification with a commercial high-electron-mobility transistor (HEMT) amplifier mounted on the $3 \mathrm{~K}$ plate, the signal is measured with a spectrum analyzer or a vector network analyzer. To prepare a cold, dissipative mechanical bath we follow the approach outlined in Ref. 18 and use optomechanical sideband cooling [23, 24] to prepare the mechanical oscillator as a strongly dissipative, cold reservoir. We proceed by pumping the auxiliary mode on the lower motional sideband (Fig. 1D). We strongly damp the mechanical oscillator to an effective energy decay rate $\Gamma_{\text {eff }} \approx 2 \pi \cdot 500 \mathrm{kHz}$ (corresponding to a mean intra-cavity photon number of $n_{\mathrm{c}}^{\text {aux }} \approx 1.5 \cdot 10^{8}$ ), while still remaining in the weak-coupling regime for the auxiliary mode. Thereby, we realize a dissipative mechanical reservoir for the primary, high-Q mode, since $\Gamma_{\text {eff }} \gg \kappa$. The effective temperature of this reservoir and its utility as a quantum resource are studied below in the manuscript.

\section{Dynamical backaction on microwave light}

We first study the modified microwave cavity susceptibility resulting from the dissipative cold reservoir, i.e. the dynamical backaction on the microwave light. The engineered bath provided by the mechanical resonator modifies the response of the electromagnetic mode when a microwave tone is applied [18. With a pump detuned by $\Delta$ from the primary microwave cavity resonance, the frequency and the decay rate of the mode shift by

$$
\delta \omega_{\mathrm{om}}=\operatorname{Re} \Sigma \quad \text { and } \quad \kappa_{\mathrm{om}}=-2 \operatorname{Im} \Sigma,
$$

called mechanical spring effect and mechanical damping, respectively. The self-energy $\Sigma$ is defined as

$\Sigma=-i g^{2}\left(\frac{1}{\Gamma_{\text {eff }} / 2+i\left(\Delta+\Omega_{\mathrm{m}}\right)}-\frac{1}{\Gamma_{\mathrm{eff}} / 2+i\left(\Delta-\Omega_{\mathrm{m}}\right)}\right)$,

where $g=g_{0} \sqrt{n_{\mathrm{c}}}$ is the effective electromechanical coupling rate enhanced by the mean intracavity photon number of the primary mode $n_{\mathrm{c}}$. This effect can be viewed as radiation pressure dynamical backaction [24, 27, 28, onto

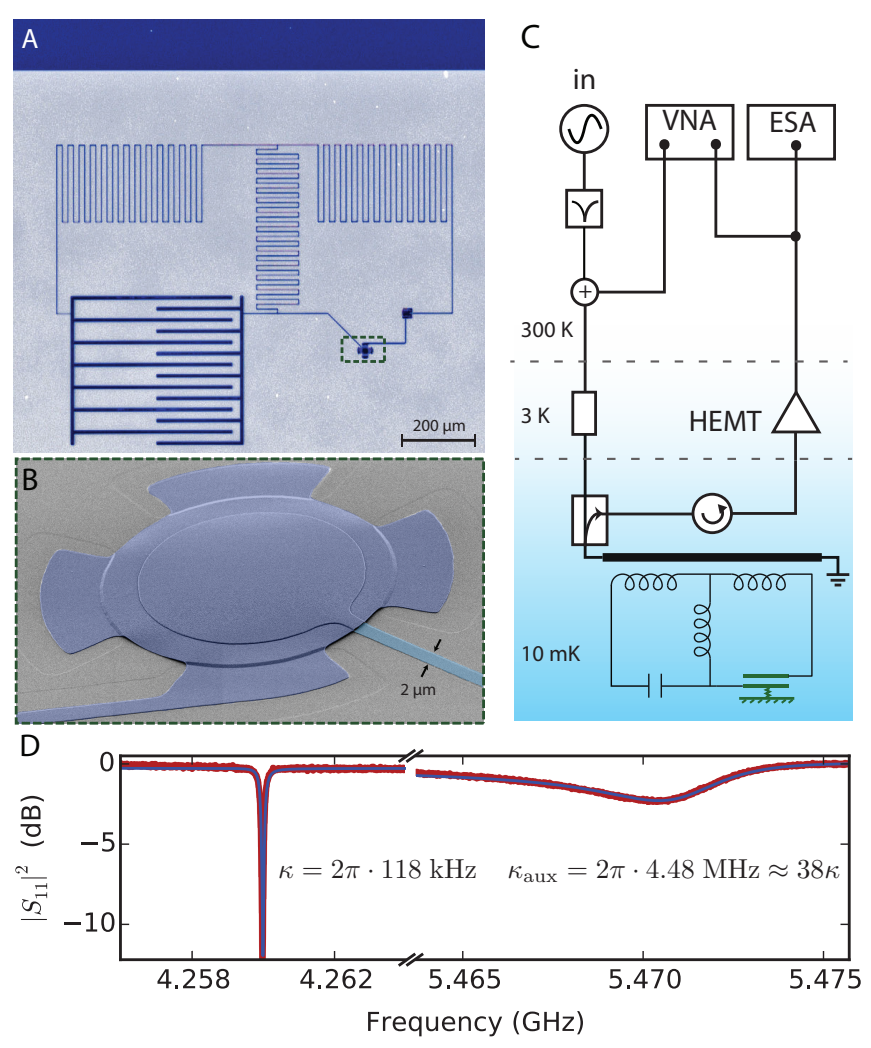

FIG. 2. Device, experimental setup, and characterization of the electromechanical circuit A. Invertedcolour optical micrograph of the circuit consisting of two coupled LC resonators, one having a mechanically compliant capacitor. Blue regions show aluminium and grey regions that are the exposed sapphire substrate. B. False-colour scanning electron micrograph of the mechanically compliant drum capacitor. C. Simplified schematics of the measurement setup with the circuit. The (multiple) input lines are filtered and attenuated at various stages before reaching the device mounted in a dilution refrigerator. Both the coherent and the spectral response can be measured. D. Linear response measurement of the device revealing the symmetric (bright, used as the auxiliary) and anti-symmetric (dark, used as the primary) microwave modes.

the microwave mode. This leads to a change in the reflection from the microwave cavity, due to a modification of its susceptibility (defined as $\hat{a}_{\text {out }}(\omega)=S_{11}(\omega) \hat{a}_{\text {in }}(\omega)$, where $\hat{a}_{\text {in,out }}(\omega)$ are Fourier domain operators associated with the input and output fields, cf. Fig. 1B). The susceptibility becomes

$$
S_{11}(\omega)=\frac{\kappa_{0}+\kappa_{\mathrm{om}}-\kappa_{\mathrm{ex}}-i 2\left(\omega-\omega_{\mathrm{c}}^{\prime}\right)}{\kappa_{0}+\kappa_{\mathrm{om}}+\kappa_{\mathrm{ex}}-i 2\left(\omega-\omega_{\mathrm{c}}^{\prime}\right)},
$$

where $\kappa_{0}$ is the internal loss of the primary mode and $\omega_{\mathrm{c}}^{\prime}=\omega_{\mathrm{c}}+\delta \omega_{\mathrm{om}}$ the modified resonance frequency.

The engineered reservoir therefore supplies a way to tailor the susceptibility of the primary electromagnetic mode, which we can directly probe using a coherent response measurement. First, we fix the detuning to either motional sideband of the primary mode, and measure 
A

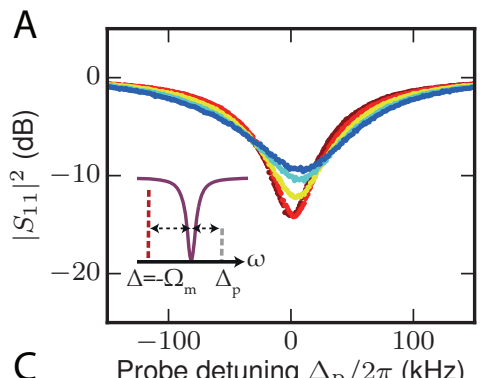

C Probe detuning $\Delta_{\mathrm{p}} / 2 \pi(\mathrm{kHz})$

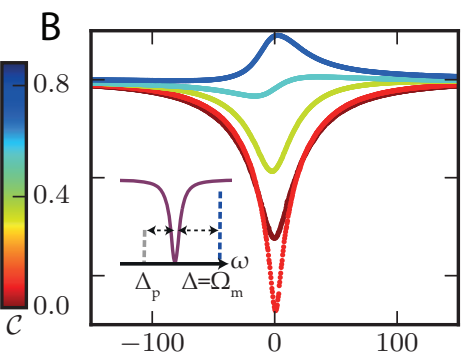

Probe detuning $\Delta_{\mathrm{p}} / 2 \pi(\mathrm{kHz})$

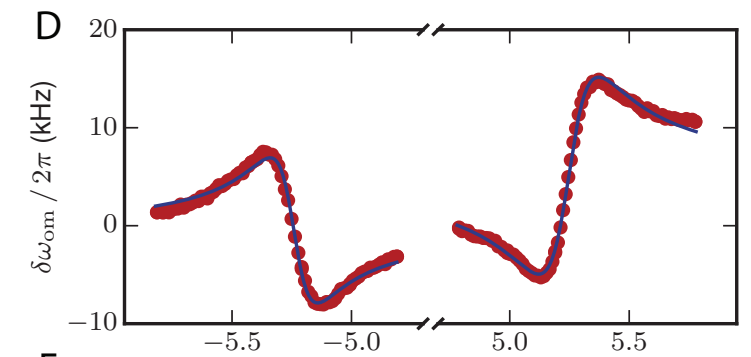

$\mathrm{E}$

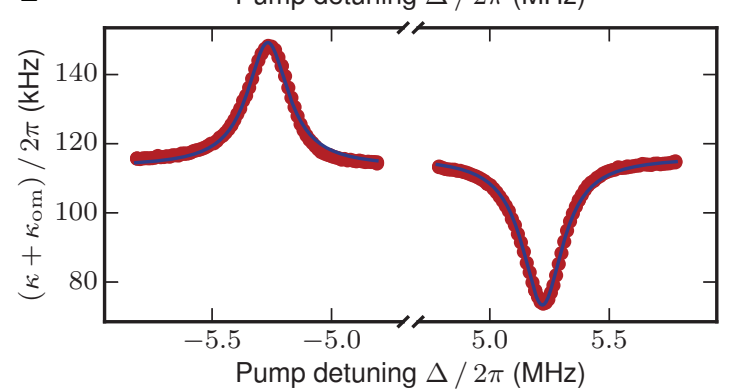

FIG. 3. Dynamical backaction on the microwave mode using an engineered mechanical reservoir. A, B. The modification of the susceptibility of the microwave cavity when a pump tone is placed on the lower and upper motional sideband of the primary mode (i.e. $\Delta=\mp \Omega_{\mathrm{m}}$ cf. inset), shown for various values of the multiphoton cooperativity $(\mathcal{C}=1$ corresponds to a mean intracavity photon number of $n_{\mathrm{c}} \approx 5 \cdot 10^{6}$ ). The slight shift of the peaks comes from the finite sideband resolution parameter of the auxiliary mode $\left(\Omega_{\mathrm{m}} / \kappa_{\text {aux }}\right)$. C. The depth of the resonance changes depending on the effective internal losses $\kappa_{0}+\kappa_{\mathrm{om}}$. Measurements with a pump on the lower and upper sideband $\left(\Delta=-\Omega_{\mathrm{m}}\right.$ for the red squares and $\Delta=+\Omega_{\mathrm{m}}$ for the blue circles), and a theoretical fit are shown. The cavity is originally undercoupled. When pumped on the upper motional sideband, it first becomes critically coupled, then overcoupled as the power of the pump tone is increased. For $\kappa_{0}+\kappa_{\mathrm{om}}<0$, there is a net gain and the electromechanical system acts as a phase-preserving microwave amplifier. D, E. Using a fixed pump power, the detuning $\Delta$ of the pump tone is swept and the change in the microwave resonance frequency and decay rate is recorded. The theoretical fit corresponds to equation (3), showing good agreement with the experimental data.

$S_{11}(\omega)$ while the power is varied. For this choice of detuning (i.e. $\Delta=\mp \Omega_{\mathrm{m}}$ ), we have $\delta \omega_{\mathrm{om}}=0$ (neglecting the term $\left.\propto \Gamma_{\text {eff }}^{2} / \Omega_{\mathrm{m}}\right)$ and the change in the microwave decay rate simplifies to

$$
\kappa_{\mathrm{om}}= \pm \mathcal{C} \kappa
$$

directly proportional to the cooperativity $\mathcal{C}=$ $4 g^{2} /\left(\kappa \Gamma_{\text {eff }}\right)$. Fig. 3A, B show the linear response for a tone on the lower and upper sideband for various pump powers. The width of the resonance, corresponding to the cavity decay rate, increases (for $\Delta=-\Omega_{\mathrm{m}}$ ) or decreases (for $\Delta=+\Omega_{\mathrm{m}}$ ) linearly with $\mathcal{C}$, accordingly. Strikingly, the depth of reflection on resonance $\left|S_{11}\left(\omega_{\mathrm{c}}\right)\right|^{2}$ varies significantly to reflect this change (Fig. $3 \mathrm{C}$ ). The effective internal loss of the cavity $\kappa_{0}+\kappa_{\text {om }}$ can be tuned on demand by changing the coupling to the dissipative reservoir via the pump tone. While the microwave cavity is initially undercoupled $\left(\kappa_{\text {ex }}<\kappa_{0}\right)$, pumping on the upper sideband reduces the effective internal loss and increases the depth on resonance until the cavity becomes critically coupled (the effective internal loss matches the external coupling, i.e. $\kappa_{0}+\kappa_{\mathrm{om}}=\kappa_{\mathrm{ex}}$ ). Increasing the power further, the cavity becomes overcoupled $\left(\kappa_{0}+\kappa_{\mathrm{om}}<\kappa_{\mathrm{ex}}\right)$ and resonant reflection increases again. When $\kappa_{0}+\kappa_{\mathrm{om}}$ becomes negative, there is net internal gain: the absorp- tive feature in the cavity reflection becomes a peak, indicating amplification of the reflected microwave signal. By pumping on the lower sideband $\left(\Delta=-\Omega_{\mathrm{m}}\right)$, extra damping is introduced and the resonance becomes increasingly undercoupled. The mechanical mode provides a cold, dissipative bath for the microwave resonator, downconverting the cavity photons to the pump. In Fig. $3 \mathrm{C}$ we plot the resonant reflection and observe good agreement with the expected dependence according to equation (5). For the data corresponding to the pump tuned to the lower motional sideband $\left(\Delta=-\Omega_{\mathrm{m}}\right)$, the depth of the resonance is systematically lower than expected, due to a decrease in the intrinsic microwave cavity loss in the presence of a strong pump [29]. In Fig. 3D, E, we keep the pump power constant and sweep the detuning $\Delta$, to measure the mechanical spring and damping effects. For the frequency shift $\delta \omega_{\text {om }}$, intrinsic non-linearities redshift the resonance frequency in an asymmetric fashion, providing a different background for the red and blue sidebands. The spring effect agrees well with the prediction from equation (3) when the two sidebands are fitted independently with different constant offsets. We note that the mechanical spring effect as a function of detuning has the opposite parity compared to the better known case of the optical spring effect [10]. 
A

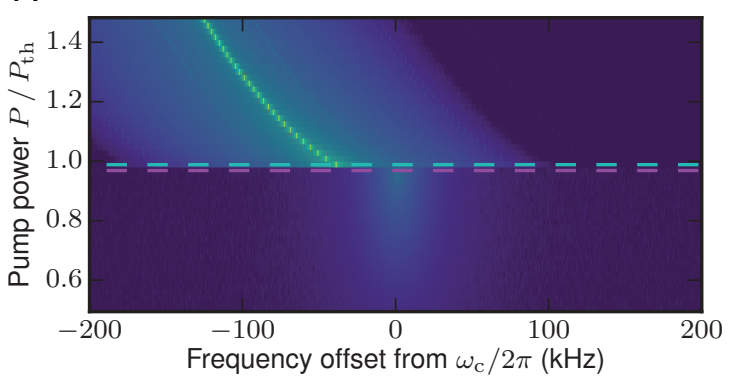

B

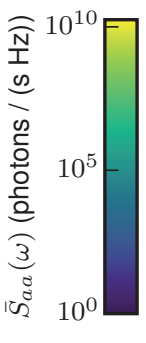

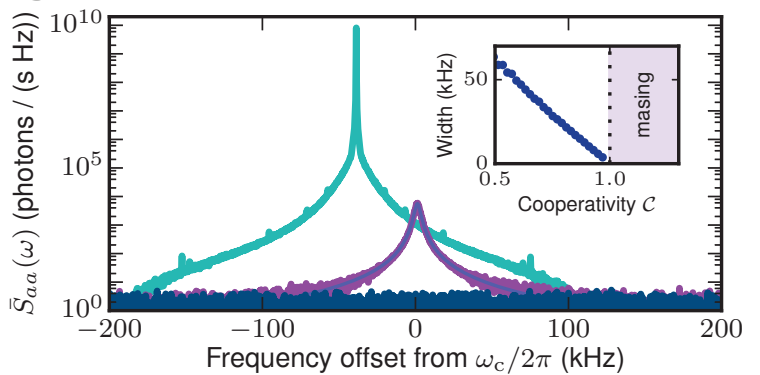

FIG. 4. Amplified vacuum fluctuations and parametric instability of the microwave mode (masing). A. Noise spectrum of the cavity emission as a function of the power of a pump on the upper motional sideband. The spectrum is measured in quanta using the HEMT amplifier for calibration (cf. main text). Above a certain threshold power $P_{\text {th }}$, the microwave mode undergoes self-sustained oscillations, characteristic to masing. The vertical axis is normalized by the pump power at masing threshold $P_{\mathrm{th}}$, equivalent to the cooperativity $\mathcal{C}=P / P_{\text {th }}$ below threshold. B. Two examples of emission from the microwave mode at the input of the HEMT, below and above masing threshold (line cuts of A), as well as a reference measurement of the background without the pump (dark blue). The inset shows the emission linewidth narrowing below threshold. In this regime, noise emission is composed of amplified vacuum and thermal fluctuations, described by equation (7). The analysis reveals that amplified vacuum noise amounts to $60 \%$ of the total, below the instability threshold.

\section{Maser action and amplification}

In the remainder of the letter, we demonstrate the cold nature of the dissipative mechanical reservoir by studying the noise properties of the system. To this end, we fix the microwave drive to the upper sideband $\left(\Delta=+\Omega_{\mathrm{m}}\right)$ and study the regime where the pump introduces net gain in the microwave cavity $\left(\kappa_{0}+\kappa_{\mathrm{om}}<0\right)$. We use a different (second) device for this analysis, with optimized properties, due to higher coupling strength $\left(g_{0}=2 \pi \cdot(106,79) \mathrm{Hz}\right.$ for the primary and auxiliary modes, respectively) and the primary mode being overcoupled $\left(\kappa_{\mathrm{ex}} / \kappa=0.76\right)$, cf. SI. In Fig. 4A, the emitted noise spectra of the microwave cavity are shown for different pump powers. The measured power spectrum is rescaled to the symmetrized cavity output field spectrum [30] $\bar{S}_{a a}(\omega)$ in units of photons per second (i.e. flux) per unit bandwidth, using the noise temperature of the HEMT as an absolute noise reference (cf. SI). As the pump compensates for the losses, the width of the emitted noise spectrum, corresponding to the cavity linewidth $\kappa_{\text {eff }}=(1-\mathcal{C}) \kappa$, decreases linearly with the pump power towards zero (at unity cooperativity $\mathcal{C}=1$ ), cf. inset of Fig. $4 \mathrm{~B}$. In this below-threshold regime, the peak photon flux spectral density emitted from the cavity increases with power, as the vacuum noise and the residual thermal microwave noise (consisting in both a finite residual occupancy $n_{\text {eff }}$ of the dissipative mechanical reservoir and a finite thermal microwave occupation of the cavity $n_{\text {cav }}$ ) are amplified according to

$\bar{S}_{a a}(\omega)=\kappa_{\mathrm{ex}} \frac{\left(\kappa_{\mathrm{ex}}-\kappa_{\mathrm{eff}}\right) \frac{1}{2}+\mathcal{C} \kappa\left(n_{\mathrm{eff}}+\frac{1}{2}\right)+\kappa_{0}\left(n_{\mathrm{cav}}+\frac{1}{2}\right)}{\left(\frac{\kappa_{\mathrm{eff}}}{2}\right)^{2}+\left(\omega-\omega_{\mathrm{c}}\right)^{2}}$

where the thermal input noise is neglected and only the amplified noise is considered. We analyze the noise prop- erties of the device in detail below when considering amplification and added noise. We find the residual thermal occupation of the dissipative reservoir to be $n_{\mathrm{eff}}=0.66$, when neglecting $n_{\text {cav }}$. Equation (7) then implies that $60 \%$ of the emitted noise from the cavity is amplified vacuum fluctuations, when $\mathcal{C} \rightarrow 1$.

For $\mathcal{C}=1$ and greater pump powers, the microwave mode undergoes self-sustained oscillations. This regime leads to a parametric instability and electromechanical maser action of the microwave mode, via stimulated emission of microwave photons into the microwave cavity. The salient features of maser action are a transition from sub- to above-threshold masing, as well as linewidth narrowing. These observations are analogues to the radiation-pressure-induced parametric instability of a mechanical mode in the normal optomechanical regime $\left(\kappa \gg \Gamma_{\mathrm{m}}\right)$ [22, 28, 31, 32. In the experiments a clear threshold behavior, characteristic of masing, is demonstrated when the emitted noise abruptly increases in strength at $\mathcal{C}=1$ (cf. Fig. $4 \mathrm{~B}$ ). Such microwave lasing in superconducting circuits has previously been demonstrated using a single artificial atom [33. Due to the strong photon population generated by masing, nonlinearities of the cavities red-shift the frequency of emission. This clearly distinguishes masing from the mechanical parametric instability (i.e. phonon lasing [32]) in the normal optomechanical regime, as in the latter case the emission does not follow the cavity but has a constant detuning of $-\Omega_{\mathrm{m}}$ with respect to the pump.

Below the masing threshold, the microwave mode coupled to the dissipative bath acts as a phase-insensitive parametric amplifier [18, 34] for incoming signals. For $\kappa_{0}+\kappa_{\text {om }}<0$, there is a net internal gain and the susceptibility $S_{11}(\omega)$ develops a peak, implying that reflection is larger than input for signals within the resonance bandwidth (in-band). The power gain of the amplifier 
A

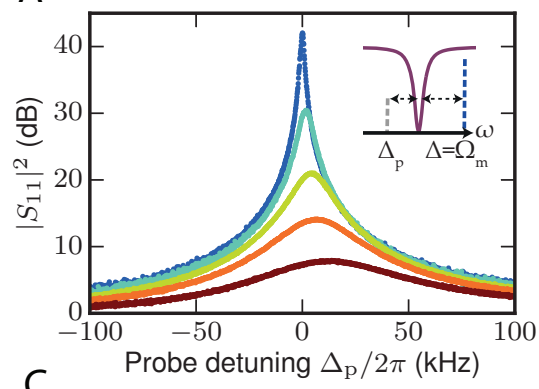

C

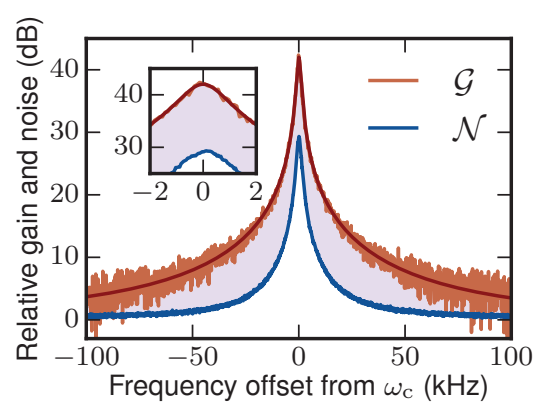

B
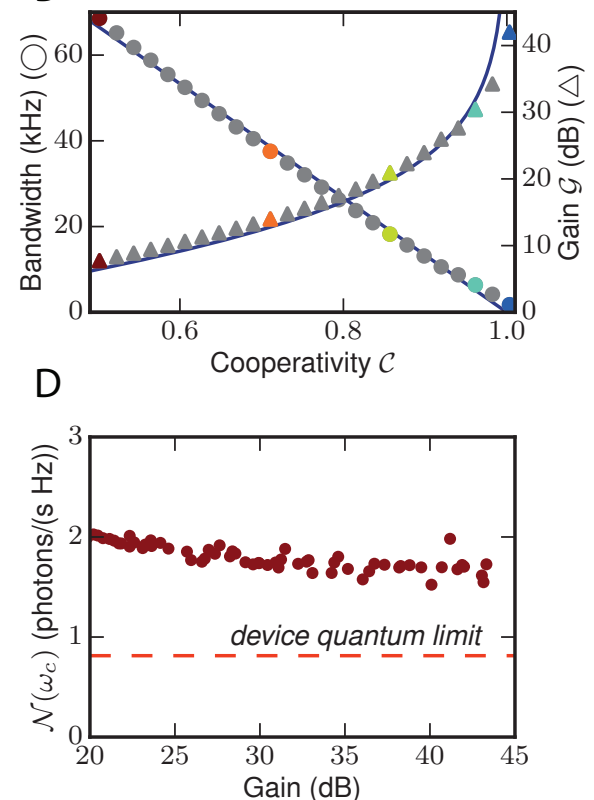

FIG. 5. Near-quantum-limited phase-preserving amplification. A. Linear response of the cavity, with increasing powers of the pump on the upper sideband from red to blue. B. Power gain (triangles) and bandwidth (circles) of the amplifier extracted from a fit of the linear response, as a function of the cooperativity of the pump on the upper sideband. The colored points correspond to the curves on panel A. C. Relative gain and noise of the amplifier, sharing the same baseline. The difference from noise to gain corresponds to over $12 \mathrm{~dB}$ of apparent signal-to-noise ratio improvement of our device over the HEMT, from which the insertion loss between the HEMT and the device (measured separately to be $1.6 \mathrm{~dB}$ ) must be subtracted to infer the real improvement. D. Added noise of the amplification referred to the input, expressed in quanta. The total added noise in the high-gain limit amounts to $1.68 \pm 0.02$ quanta, corroborated by an independent optomechanical calibration technique (cf. SI). This is only 0.87 quanta above the device quantum limit $n_{\mathrm{DQL}}$, defined in equation 10 .

is defined as the resonance peak height above the background, given by (cf. SI)

$$
\mathcal{G}\left(\omega_{\mathrm{c}}\right)=\left|S_{11}\left(\omega_{\mathrm{c}}\right)\right|^{2}=\left|\frac{\left(2 \frac{\kappa_{\mathrm{ex}}}{\kappa}-1\right)+\mathcal{C}}{1-\mathcal{C}}\right|^{2} .
$$

The bandwidth of the amplifier is the linewidth of the microwave resonance, given by $\kappa_{\text {eff }}=(1-\mathcal{C}) \kappa$. In order to measure the gain, bandwidth and noise properties of the amplifier, we inject, in addition to the pump tone on the upper sideband $\left(\Delta=+\Omega_{\mathrm{m}}\right)$, a weak signal tone (swept around the cavity resonance) and measure the reflected signal as a function of the pump tone power. With increasing pump power, a narrowing of the cavity bandwidth (Fig. 5A) is observed, as well as an increase in the power of the reflected signal (i.e. gain). By fitting the reflected power as a function of detuning, the gain and bandwidth as a function of cooperativity are extracted, and found to be in good agreement with the theoretical predictions given by equation (8) (Fig. 5B). The observed gain exceeds $42 \mathrm{~dB}$.

\section{E. Near-quantum-limited amplification}

Next, we study the added noise of the dissipative amplification process. The added noise $\mathcal{N}$, as referred to the input of the amplifier, is given by the noise output of equation (7) without the input noise and divided by the gain $\mathcal{G}\left(\omega_{\mathrm{c}}\right)$. On resonance, it is found to be (cf. SI)

$$
\mathcal{N}\left(\omega_{\mathrm{c}}\right)=\frac{4 \mathcal{C} \frac{\kappa_{\mathrm{ex}}}{\kappa}\left(n_{\mathrm{eff}}+\frac{1}{2}\right)+4 \frac{\kappa_{\mathrm{ex}} \kappa_{0}}{\kappa^{2}}\left(n_{\mathrm{cav}}+\frac{1}{2}\right)}{\left(\mathcal{C}-1+\frac{2 \kappa_{\mathrm{ex}}}{\kappa}\right)^{2}},
$$

which, in the high gain limit $(\mathcal{C} \rightarrow 1)$, simplifies to $\mathcal{N}\left(\omega_{c}\right) \rightarrow \frac{\kappa_{0}}{\kappa_{\text {ex }}}\left(n_{\text {cav }}+\frac{1}{2}\right)+\frac{\kappa}{\kappa_{\text {ex }}}\left(n_{\text {eff }}+\frac{1}{2}\right)$. This quantity can be measured by recording the improvement of the signal-to-noise ratio (SNR) of amplification in and out of the bandwidth of our device. This directly compares the noise performance of our device with the commercial HEMT amplifier, which is used as a calibrated noise source (the noise temperature of the HEMT is measured separately at $\omega_{\mathrm{c}}$ and found to be $3.95 \pm 0.02 \mathrm{~K}$, corresponding to $20.0 \pm 0.1$ quanta, cf. SI). In Fig. 5 C, the gain of the device is compared to the noise output of the chain, normalized to the HEMT noise background. This calibration was corroborated by a second, independent calibration technique, which uses the scattered power in the motional sideband in conjunction with the knowledge 
of the intracavity photon number and $g_{0}$ (cf. SI). The relative gain of the signal exceeds the relative noise by over $12 \mathrm{~dB}$. From this apparent SNR improvement, one must subtract the insertion loss of the components between the device and the HEMT, measured independently at $77 \mathrm{~K}$ to be $1.6 \mathrm{~dB}$ (cf. SI). The analysis reveals therefore that the optomechanical amplifier provides more than 10 $\mathrm{dB}$ of improvement over the SNR of the HEMT. The inferred added noise on resonance is shown as a function of gain in Fig. $5 \mathrm{D}$; in the high-gain limit, it is a constant value of $\mathcal{N}\left(\omega_{\mathrm{c}}\right)=1.68 \pm 0.02$ quanta per second per unit bandwidth (with the uncertainty given by statistical fluctuations). Using equation (9) and assuming $n_{\text {cav }}=0$, the effective occupation of the dissipative reservoir is found to be $n_{\text {eff }}=0.66$. However, the strong cooling pump increases the temperature of the cavity thermal bath to an occupation $n_{\text {cav }}=1.03$, obtained from measuring the emitted thermal noise of the microwave cavity. (cf. SI). Taking the residual cavity thermal noise into account, the estimate for the mechanical occupation is reduced to $n_{\text {eff }}=0.41$. This demonstrates that the dissipative mechanical reservoir constitutes a quantum resource. We note that even in the case when all the thermal noise sources are reduced to zero (i.e. $n_{\text {eff }}=n_{\text {cav }}=0$ ), the added noise of the amplifier is

$$
n_{\mathrm{DQL}}=\frac{1}{2}+\frac{\kappa_{0}}{\kappa_{\mathrm{ex}}}
$$

which, we call the device quantum limit and deviates from $1 / 2$ due to the finite internal dissipation rate $\kappa_{0}$. For the present system the device quantum limit amounts to 0.81 quanta for the coupling ratio of $\kappa_{\mathrm{ex}} / \kappa=0.76$, which is only 0.87 quanta below the added noise we measure. Compared to other electromechanical phase-preserving amplifiers 35, 36, the preparation of an engineered cold, dissipative mechanical bath enables lower added noise. It is interesting to compare the present amplifier scheme, relying on a dissipative reservoir, to the microwave parametric amplifiers as used in circuit QED. In the latter case, typically both idler and signal are resonant with one or more microwave cavities 37 39. As gain increases, this leads to a simultaneous increase in both the signal and idler mode population. In contrast, while the present amplifier scheme uses a parametric interaction as well, the large dissipation rate for the (mechanical) idler mode only leads to the generation of a signal photon (microwave field), suppressing the idler, a situation akin to a Raman-type interaction found in nonlinear optics [40].

\section{F. Conclusions}

In summary, we have implemented and studied a new regime of circuit electromechanics by coupling an electromagnetic cavity mode to an engineered cold dissipative reservoir formed by a mechanical oscillator. The usual roles of the two modes are reversed, allowing for dynamical backaction on the microwave mode using the mechan- ical reservoir. We demonstrate the control of the internal losses of the cavity in the form of backaction-induced amplification, de-amplification, and masing of the microwave field. By performing microwave amplification close to the quantum limit, we show that the mechanical reservoir functions as a useful quantum resource.

The near-quantum-limited amplification with a mechanical reservoir extends the available quantum information manipulation toolkit, adding to the existing devices based on Josephson junctions [37 39, 41]. While the present amplifier is not frequency-tunable, recent advances in circuit electromechanics have demonstrated such functionality 42. The observed reservoir-mediated microwave damping may allow to remove residual thermal occupancy from the microwave cavity, akin to cooling schemes developed in circuit QED 43. Moreover, the control over internal dissipation enables allelectromechanical tuning of the coupling of the microwave resonator to the feedline, offering the potential for an electromechanical reconfigurable network [44. While the present scheme employs a single pump tone, dual tone pumping would lead to the preparation of squeezed states of the microwave cavity [19]. Viewed more broadly, the realization of a cold mechanical reservoir for microwave light provides a central ingredient for novel electromechanical devices. Indeed, the circuit can be extended to multiple microwave resonators coupled to a shared mechanical reservoir and implement the dissipative cavity-cavity interactions that are at the heart of recent schemes to entangle microwave photons [16] and, combined with coherent interactions, to perform nonreciprocal microwave transmission 20. Such nonreciprocal devices can be of use for the rapidly expanding field of circuit QED [45, 46].

\section{Data availability statement}

The data that support the plots within this paper and other findings of this study are available from the corresponding author upon reasonable request.

\section{Author contributions}

T.J.K. and A.K.F. conceived the idea. L.D.T. fabricated the devices. L.D.T. and N.R.B., under the supervision of A.K.F., performed the measurements. N.R.B. carried out the data analysis. A.N. contributed to the theoretical framework. All authors contributed to writing the manuscript.

\section{ACKNOWLEDGMENTS}

We thank A. A. Clerk, V. Sudhir and D. Wilson for helpful comments, E. Glushkov for helping out with the 
measurement setup and C. Javerzac-Galy for general assistance. This work was funded by the SNF, the NCCR Quantum Science and Technology (QSIT), the European Union Seventh Framework Program through iQUOEMS (grant no. 323924). LDT is supported by Marie Curie ITN cQOM (grant no. 290161). TJK acknowledges fi- nancial support from an ERC AdG (QuREM). AN holds a University Research Fellowship from the Royal Society and acknowledges support from the Winton Programme for the Physics of Sustainability. All samples were fabricated in the Center of MicroNanoTechnology (CMi) at EPFL.
[1] A. O. Caldeira and A. J. Leggett, Phys. Rev. Lett. 46, 211 (1981)

[2] J. F. Poyatos, J. I. Cirac, and P. Zoller, Physical Review Letters 77, 4728 (1996).

[3] H. Krauter, C. A. Muschik, K. Jensen, W. Wasilewski, J. M. Petersen, J. I. Cirac, and E. S. Polzik, Phys. Rev. Lett. 107, 080503 (2011).

[4] J. T. Barreiro, M. Muller, P. Schindler, D. Nigg, T. Monz, M. Chwalla, M. Hennrich, C. F. Roos, P. Zoller, and R. Blatt, Nature 470, 486 (2011)

[5] Y. Lin, J. P. Gaebler, F. Reiter, T. R. Tan, R. Bowler, A. S. Sørensen, D. Leibfried, and D. J. Wineland, Nature 504, 415 (2013)

[6] D. Kienzler, H.-Y. Lo, B. Keitch, L. d. Clercq, F. Leupold, F. Lindenfelser, M. Marinelli, V. Negnevitsky, and J. P. Home, Science 347, 53 (2015).

[7] K. W. Murch, U. Vool, D. Zhou, S. J. Weber, S. M. Girvin, and I. Siddiqi, Physical Review Letters 109, $183602(2012)$

[8] S. Shankar, M. Hatridge, Z. Leghtas, K. M. Sliwa, A. Narla, U. Vool, S. M. Girvin, L. Frunzio, M. Mirrahimi, and M. H. Devoret, Nature 504, 419 (2013).

[9] Z. Leghtas, S. Touzard, I. M. Pop, A. Kou, B. Vlastakis, A. Petrenko, K. M. Sliwa, A. Narla, S. Shankar, M. J. Hatridge, M. Reagor, L. Frunzio, R. J. Schoelkopf, M. Mirrahimi, and M. H. Devoret, Science 347, 853 (2015).

[10] M. Aspelmeyer, T. J. Kippenberg, and F. Marquardt, Reviews of Modern Physics 86, 1391 (2014)

[11] A. Kronwald, F. Marquardt, and A. A. Clerk, Physical Review A 88, 063833 (2013).

[12] M. J. Woolley and A. A. Clerk, Physical Review A 89, $063805(2014)$

[13] E. E. Wollman, C. U. Lei, A. J. Weinstein, J. Suh, A. Kronwald, F. Marquardt, A. A. Clerk, and K. C. Schwab, Science 349, 952 (2015)

[14] J.-M. Pirkkalainen, E. Damskägg, M. Brandt, F. Massel, and M. Sillanpää, Physical Review Letters 115, 243601 (2015)

[15] F. Lecocq, J. Clark, R. Simmonds, J. Aumentado, and J. Teufel, Physical Review X 5, 041037 (2015).

[16] Y.-D. Wang and A. A. Clerk, Physical Review Letters 110, 253601 (2013)

[17] A. Metelmann and A. Clerk, Physical Review Letters 112, 133904 (2014)

[18] A. Nunnenkamp, V. Sudhir, A. K. Feofanov, A. Roulet, and T. J. Kippenberg, Physical Review Letters 113, $023604(2014)$

[19] A. Kronwald, F. Marquardt, and A. A. Clerk, New Journal of Physics 16, 063058 (2014)

[20] A. Metelmann and A. Clerk, Physical Review X 5, $021025(2015)$
[21] J. D. Teufel, D. Li, M. S. Allman, K. Cicak, A. J. Sirois, J. D. Whittaker, and R. W. Simmonds, Nature 471, 204 (2011).

[22] V. Braginsky and A. Manukin, Measurement of weak forces in Physics experiments (Univ. of Chicago Press, 1977).

[23] J. D. Teufel, J. W. Harlow, C. A. Regal, and K. W. Lehnert, Phys. Rev. Lett. 101, 197203 (2008)

[24] A. Schliesser, R. Rivière, G. Anetsberger, O. Arcizet, and T. J. Kippenberg, Nat Phys 4, 415 (2008).

[25] K. Cicak, D. Li, J. A. Strong, M. S. Allman, F. Altomare, A. J. Sirois, J. D. Whittaker, J. D. Teufel, and R. W. Simmonds, Applied Physics Letters 96, 093502 (2010).

[26] J. M. Dobrindt, I. Wilson-Rae, and T. J. Kippenberg, Phys. Rev. Lett. 101, 263602 (2008)

[27] V. Braginsky, A. Manukin, and M. Y. Tikhonov, Sov. Phys. JETP 31, 829 (1970).

[28] T. J. Kippenberg, H. Rokhsari, T. Carmon, A. Scherer, and K. J. Vahala, Phys. Rev. Lett. 95, 033901 (2005)

[29] A. Megrant, C. Neill, R. Barends, B. Chiaro, Y. Chen, L. Feigl, J. Kelly, E. Lucero, M. Mariantoni, P. J. J. O'Malley, D. Sank, A. Vainsencher, J. Wenner, T. C. White, Y. Yin, J. Zhao, C. J. Palmstrøm, J. M. Martinis, and A. N. Cleland, Applied Physics Letters 100, 113510 (2012).

[30] A. A. Clerk, M. H. Devoret, S. M. Girvin, F. Marquardt, and R. J. Schoelkopf, Reviews of Modern Physics 82, 1155 (2010).

[31] F. Marquardt, J. G. E. Harris, and S. M. Girvin, Phys. Rev. Lett. 96, 103901 (2006).

[32] I. S. Grudinin, H. Lee, O. Painter, and K. J. Vahala, Phys. Rev. Lett. 104, 083901 (2010)

[33] O. Astafiev, K. Inomata, A. O. Niskanen, T. Yamamoto, Y. A. Pashkin, Y. Nakamura, and J. S. Tsai, Nature 449, 588 (2007)

[34] C. M. Caves, Physical Review D 26, 1817 (1982)

[35] F. Massel, T. T. Heikkilä, J. M. Pirkkalainen, S. U. Cho, H. Saloniemi, P. J. Hakonen, and M. A. Sillanpää, Nature 480, 351 (2011)

[36] C. F. Ockeloen-Korppi, E. Damskägg, J.-M. Pirkkalainen, T. T. Heikkilä, F. Massel, and M. A. Sillanpää, Physical Review X 6, 041024 (2016)

[37] N. Bergeal, F. Schackert, M. Metcalfe, R. Vijay, V. E. Manucharyan, L. Frunzio, D. E. Prober, R. J. Schoelkopf, S. M. Girvin, and M. H. Devoret, Nature 465, 64 (2010)

[38] C. Eichler, Y. Salathe, J. Mlynek, S. Schmidt, and A. Wallraff, Phys. Rev. Lett. 113, 110502 (2014)

[39] M. A. Castellanos-Beltran, K. D. Irwin, G. C. Hilton, L. R. Vale, and K. W. Lehnert, Nat Phys 4, 929 (2008)

[40] N. Bloembergen, Nonlinear Optics, 4th ed. (World Scientific Pub Co Inc, 1996).

[41] K. M. Sliwa, M. Hatridge, A. Narla, S. Shankar, L. Frunzio, R. J. Schoelkopf, and M. H. Devoret, Phys. Rev. X 
5, $041020(2015)$

[42] R. W. Andrews, A. P. Reed, K. Cicak, J. D. Teufel, and K. W. Lehnert, Nature Communications 6, 10021 (2015)

[43] M. Grajcar, S. H. W. van der Ploeg, A. Izmalkov, E. Il'ichev, H. G. Meyer, A. Fedorov, A. Shnirman, and G. Schön, Nat Phys 4, 612 (2008)
[44] J. Kerckhoff, R. W. Andrews, H. S. Ku, W. F. Kindel, K. Cicak, R. W. Simmonds, and K. W. Lehnert, Phys. Rev. X 3, 021013 (2013)

[45] A. Wallraff, D. I. Schuster, A. Blais, L. Frunzio, R. S. Huang, J. Majer, S. Kumar, S. M. Girvin, and R. J. Schoelkopf, Nature 431, 162 (2004)

[46] M. H. Devoret and R. J. Schoelkopf, Science 339, 1169 (2013). 